\section{New Avian Model of Experimental Glomerulonephritis Consistent with Mediation by Cellular Immunity Nonhumorally Mediated Glomerulonephritis in Chickens}

W. Kline Bolton, F. Lee Tucker, and Benjamin C. Sturgill Department of Internal Medicine and Department of Pathology, University of Virginia School of Medicine,

Charlottesville, Virginia 22908 bstract. The present study examined the role of cell-mediated immunity (CMI) in the production of experimental autoimmune glomerulonephritis (EAG) in chickens deficient in humorally mediated immunity (HMI). Cyclophosphamide bursectomized (Bsx) and normal control chickens were used. Bsx chickens were used only if they had severe depression of HMI, which was evidenced by marked reduction in bursal weights $(0.89 \pm 0.23$ vs. $2.92 \pm 0.9 \mathrm{~g})$, decreased serum $\mathrm{IgG}$ to $\leqslant 10 \%$ of normal, and total lack of HMI to immunization with sheep red blood cells. EAG was produced by immunizing chickens with bovine glomerular basement membrane (GBM) in complete Freund's adjuvant. CMI manifested by wattle thickness increments to PPD was not different, $3.89 \pm 0.45 \mathrm{~mm}$ for Bsx compared with $3.73 \pm 0.75 \mathrm{~mm}$ for controls. No circulating antibodies to GBM developed in $68 \%$ of Bsx chickens, and the anti-GBM titers were $<1: 312$ in those Bsx chickens positive for antibody compared with $>2,000$ for controls. GBM deposits of $\mathrm{IgG}$ by fluorescence were much decreased, $0.53 \pm 0.16$ compared with $2.19 \pm 0.32$ for controls, and were absent in $64 \%$ of Bsx chickens. Nonetheless, proliferative nephritis with crescents was present and was even more severe in Bsx

Portions of this work were presented at the Annual Meetings of the Southern Society for Clinical Investigation in New Orleans, January 1982, and at the International Academy of Pathology, Boston, March 1982, and have been published in abstract form in 1981, Clin. Res. 29:868, and in 1982, Lab. Invest. 46:84.

Dr. Tucker is the recipient of a Southern Medical Associatioin grant. Address correspondence and reprint requests to Dr. Bolton.

Received for publication 25 May 1982 and in revised form 10 January 1984

J. Clin. Invest.

(c) The American Society for Clinical Investigation, Inc. 0021-9738/84/05/1263/14 $\$ 1.00$

Volume 73, May 1984, 1263-1276 chickens than in controls, with glomerular sizes of $20.8 \pm 0.6 \mathrm{U}$ for Bsx-GBM, 19.8 \pm 1.2 for control-GBM, $14.9 \pm 1.5$ for Bsx, and $13.6 \pm 0.8$ for normal chickens. Nephritic eluates did not produce disease in normal chickens, while administration of sensitized cells with $\left[\mathrm{H}^{3}\right]$ thymidine to naive birds was associated with increased mesangial grain counts by autoradiography.

These findings suggest that CMI plays a major role in the pathogenesis of EAG in chickens in the absence of HMI. By implication, CMI may be crucial in the development of other types of glomerulonephritis as well.

\section{Introduction}

Glomerulonephritis has classically been defined as an inflammatory process involving the glomerulus, one usually mediated by the deposition of immune complexes or anti-glomerular basement membrane (GBM) ${ }^{1}$ antibodies $(1,2)$. Although sensitization of the cellular immune system at immunization was assumed in the past, no pathogenetic role was ascribed to cellmediated immunity (CMI) (3). Increasing evidence, however, indicates an important role for $\mathrm{CMI}$ in the pathogenesis of many human and experimental animal types of nephritis. The macrophage appears to play a critical part in the proliferation and GBM damage in certain forms of nephritis (4-16); lymphocytes may participate in yet other mechanisms of immunologic damage $(17,18)$; and CMI of uncertain type may be important in still other forms of nephritis (19-27).

The experimental models implicating CMI in the pathogenesis of nephritis have relied upon a humoral mechanism to plant immunoglobulins in the glomerulus with consequent recruitment of exogenous cells, stimulation of endogenous resident

1. Abbreviations used in this paper: Bsx, bursectomized; CMI, cell-mediated immunity; CFA, complete Freund's adjuvant; EAG, experimental autoimmune glomerulonephritis; GBM, glomerular basement membrane; H\&E, hematoxylin and eosin; HMI, humorally mediated immunity; KFAb, kidney-fixing antibody; PAS, periodic acid Schiff's reagent; SRBC, sheep red blood cells. 
cells, and development of nephritis. Conclusions regarding the role of monocytes, macrophages, and lymphocytes have been based upon studies that focused upon migration patterns and identification of various types of cells in tissue sections, and upon effects of specific antisera to certain cell subpopulations. Nonetheless, these various investigations have been performed within the milieu of an intact humoral immune system. This factor complicates evaluation of the isolated role of CMI in the development of glomerulonephritis in the absence of glomerular immunoglobulin deposition. Indeed, there are no immunological models of glomerulonephritis induced without the participation of immunoglobulin deposits.

We have recently shown that functional $T$ cells are required for the development of anti-GBM nephropathy in a murine model $(28,29)$, and more recently demonstrated that a proliferative anti-GBM nephritis could be induced in chickens (30). Our development of this latter model has allowed us to separate the effects of the humoral immune system from CMI to further study the development of the glomerulonephritis in chickens. The results show that glomerulonephritis develops in animals with normal intact CMI, with partly impaired, and apparently absent humoral immunity to GBM. These findings suggest that the nephritis in this model may be mediated by CMI in the absence of humoral immunity or by other mechanisms that have not been elucidated. This dissociation of the development of nephritis from the humoral immune system implicates a previously undescribed process or processes in the pathogenesis of experimental glomerulonephritis in this model.

\section{Methods}

\section{Phase I}

Chickens. 100 neonatal J. J. Warren chicks 2-20-h-old were obtained from the hatchery and placed in an incubator in a laminar flow room. These animals were used in the first phase of studies that examined induction of nephritis in normal and humorally deficient birds. Animals were handled with gloves and masks, received autoclaved food, and autoclaved, acid-treated water $(0.6 \mathrm{ml}$ of $12 \mathrm{~N} \mathrm{HCl}$ per gallon). The utmost care was always taken not to expose animals to environmental viral or bacterial infections. All utensils and cages were steam cleaned weekly and the same Vivarium technician assumed the care of the animals throughout the study.

Bursectomy (Bsx). 75 chicks received $2 \mathrm{mg}$ cyclophosphamide in $0.1 \mathrm{ml}$ volume of sterile water in the thigh muscle within $20 \mathrm{~h}$ of hatching (31-33). This dose was repeated daily for $4 \mathrm{~d}$ total. The 25 cohorts did not receive cyclophosphamide and served as controls.

Immunoglobulin levels. At 5 wk of age, all chickens were bled from the wing veins and serial dilutions of sera were examined by double diffusion in micro-Ouchterlony plates using monospecific rabbit antichicken IgG (Miles Laboratories Inc., Elkhart, IN). Plates were examined at 24 and $48 \mathrm{~h}$, washed, dried, and stained with Coomassie Blue. Titers are expressed as the reciprocal of the dilutions. Selected sera from Bsx birds were also examined by immunoelectrophoresis in $1 \%$ agarose, $\mathrm{pH}$ 8.6, for $90 \mathrm{~min}$ with rabbit anti-whole chicken serum to assess levels of IgM.

Immunization with sheep red blood cells (SRBC). To evaluate the ability of the chickens to respond to an antigenic stimulus, animals were immunized with SRBC (34). These were prepared by collecting SRBC in Alsevers solution, washing them three times in sterile phosphatebuffered saline (PBS), pH 7.4, at $4^{\circ} \mathrm{C}$, and resuspending them in sterile PBS to a total cell volume of $20 \%$. Three sets of birds were immunized intraperitoneally with $0.5 \mathrm{ml}$ of $20 \%$ SRBC twice at 1 -wk intervals. The three groups were normal controls, Bsx chickens with IgG titers $\leqslant 1: 16$, and Bsx chickens with IgG titers $\geqslant 1: 32$. Animals were bled 1 wk after each injection and the sera examined for anti-SRBC antibody by hemagglutination. All sera were absorbed five times with washed SRBC (1:10 vol) at $4^{\circ} \mathrm{C}$ to absorb naturally occurring hemolysin, heat inactivated at $56^{\circ} \mathrm{C}$ for $30 \mathrm{~min}$, and divided into two portions. One portion was incubated with a vol/vol of $0.2 \mathrm{M} \mathrm{2-mercaptoethanol} \mathrm{at} 37^{\circ} \mathrm{C}$ for 30 min to inactivate IgM, and the other was used only after decomplementing. Serial dilutions of each were made in microtiter plates using $2.5 \% \mathrm{vol} / \mathrm{vol} \mathrm{SRBC}$ in PBS, $\mathrm{pH}$ 7.4. Cells and test sera were incubated at $23^{\circ} \mathrm{C}$ for $2 \mathrm{~h}$ and agglutination determined.

Preparation of bovine GBM. Normal and Bsx chickens selected as low responders to SRBC with low IgG levels were immunized with bovine GBM. Beef kidneys were obtained from a local abattoir and frozen at $-50^{\circ} \mathrm{C}$ until use. They were then thawed at $4^{\circ} \mathrm{C}$ in PBS and the cortices separated from the medullae. Minced cortices were pressed through sieves, 40,80 , and 140 mesh, respectively, to collect whole glomeruli on a 200 -mesh sieve $(28,29,35)$. Extensive washing in PBS, $4^{\circ} \mathrm{C}$, was carried out at each step. Glomerular preparations were examined by phase-contrast microscopy to ascertain degree of tubular contamination. Preparations used to immunize animals were $95 \%$ pure glomeruli. The glomeruli were washed in deionized water, lyophilized, and stored.

Immunization with $G B M$. Chickens were immunized with lyophilized bovine glomeruli, $10 \mathrm{mg}$ dry wt/bird, resuspended in sterile PBS at $4^{\circ} \mathrm{C}$, emulsified in complete Freund's adjuvant H37Ra (CFA) (Difco Laboratories, Inc., Detroit, MI), 1:1 vol/vol, and given in a 1-ml final volume subcutaneously in the axilla. Immunizations were begun at 9 wk of age and continued every other week until termination of the experiment. Both normal and Bsx chickens were immunized with GBM.

Control immunizations. Bsx and normal chickens were injected using the same schedule and method as birds receiving GBM. However, control birds received PBS in CFA without any GBM.

Tissue. Animals from the four study groups were serially killed at $5,7,9,11,13,15,17$, and 19 wk after the beginning of immunizations. At the time of sacrifice the bursa or bursal remnant was dissected free, weighed, and fixed in $10 \%$ buffered formalin. Kidney tissue was divided into three portions. One part was fixed in $10 \%$ buffered formalin for light microscopy, the second snap-frozen in dry ice isopentane for immunofluorescence, and the third fixed in formol-sucrose/gum-sucrose for nonspecific esterase staining (36-38). Animals were sacrificed by exsanguination with ether and/or ketamine anesthesia.

\section{Pathology}

a) Light microscopy. Formalin-fixed tissue was paraffin embedded and serial sections $3 \mu \mathrm{m}$ in thickness was stained with periodic acid Schiff's reagent (PAS) and hematoxylin eosin (H\&E) (36). Bursal tissue was examined for normal morphology, lymphoid tissue, follicles, and germinal centers. Kidneys were assessed qualitatively without knowledge of the study group for degree of proliferation, tubular atrophy, crescent formation, and interstitial infiltrate. Glomerular grading was on a 0-4+ basis, with notation of focal or diffuse proliferation. Proliferation was quantitatively assessed using a Zeiss eye-piece micrometer and by measuring the diameter of glomerular tufts in 10-20 glomeruli cut through the stalk (30). Data are expressed as micrometer units.

b) Immunofluorescence microscopy. Immunofluorescent examina- 
tion of kidney biopsies was performed using commercial monospecific fluorescein-labeled goat anti-chicken IgG, albumin, and IgG-A-M (Cappel Laboratories Inc., Cochranville, PA). We raised anti-fibrinogen antiserum in rabbits by immunization with fibrin that was obtained by dialyzing heparinized plasma against PBS to form a clot, by washing extensively, and by lyophilizing. $1 \mathrm{mg}$ of fibrin was given subcutaneously in CFA every other week. The resultant antiserum was absorbed with whole chicken serum and fluorescein labeled (36). While a single line was detectable against chicken plasma, no line was seen against serum. Antiserum was prepared against chicken complement attached to zymosan beads (39). The antiserum was fluorescein labeled and absorbed with cobra venom factor-depleted chicken serum. A single line was present by radial immunodiffusion in dilutions of 1:2-1:30 against normal chicken serum, but not against depleted serum. A bimodal line was detected in immunoelectrophoresis against fresh chicken serum, and fixed chicken complement was detected using the indirect assay with antinuclear factor applied to rat liver with fresh chicken serum as the complement source. Heating the chicken serum abolished fixation. An additional line nonidentical to albumin, IgG, IgM, IgA, or fibrinogen by immunodiffusion was present in neat antiserum only. Sections were read with a Zeiss research microscope with the IV FI epifluorescence condenser equipped with an $\mathrm{HBO} 50$-W super-pressure mercury lamp. A 490-nm excitation filter and a 515-nm barrier filter were used with $\times 8$ eye pieces and Planapochromat lenses. Deposits in chicken kidney were graded on an 11-point scale without knowledge of the immunization state of the animal, and described as linear or granular (38). By this method, tissue sections are given a numerical score based upon the intensity of fluorescent deposits on a $0-4+$ scale, such that negative is 0 , negative-trace is 0.25 , trace is $0.5,1+$ is $1.0,1-2+$ intensity is 1.5 , $2+$ is $2.0,2-3+$ is 2.5 , etc.

c) Nonspecific esterase staining. Kidney tissue fixed in formol-sucrose/gum-sucrose was embedded in paraffin, and 4- $\mu \mathrm{m}$ sections cut $(38,40)$. Deparaffinized sections were washed in deionized water, air dried, and incubated in pararosanilin/alpha-naphthyl acetate/sodium nitrite, $\mathrm{pH}$ 6.0, mixed immediately before incubation. Washed sections were counterstained with $1 \%$ methyl green, washed, mounted, and examined for esterase-positive cells in the glomeruli and crescents. Staining of proximal tubular cytoplasm and intravascular monocytes served as an internal control.

Anti-GBM assay. Sera obtained at sacrifice were evaluated for antiGBM antibodies. Two types of assays were used to assess circulating antibody response to bovine and chicken GBM. In the first, serial dilutions of chicken serum were examined by indirect immunofluorescence using fluoresceinated anti-chicken IgG and normal bovine and chicken kidneys (28). The second assay system was used to provide quantitative information about the degree of immune responsiveness to the bovine GBM. Collagenase-solubilized GBM was prepared by mixing lyophilized bovine glomeruli $(100 \mathrm{mg})$ in Tris $\mathrm{HCl}$ buffer, $\mathrm{pH} 7.4$, with $0.01 \mathrm{M} \mathrm{CaCl}_{2}$ and $6.4 \mathrm{mg}$ collagenase (Sigma Chemical Co., St. Louis, MO) at $37^{\circ} \mathrm{C}$ for $22 \mathrm{~h}$, and by dialyzing the supernatant against deionized water (23). The solubilized GBM $(10 \mathrm{mg})$ was attached to $\mathrm{SRBC}$ via $\mathrm{CrCl}_{3}(5 \mathrm{ml})$ for $30 \mathrm{~min}$ at $23^{\circ} \mathrm{C}$, was washed, serial dilutions of sera were absorbed with SRBC and heat inactivated as described above, and then were mixed with a $1 \%$ suspension of coated cells in microtiter plates. Hemagglutination titers are expressed as the reciprocal.

Wattle thickness assay for CMI. The wattle is the fleshy appendage hanging down from the throat or chin of the chicken. The Mycobacterium tuberculosis $\mathrm{H} 3^{7} \mathrm{Ra}$ used to immunize the animals contains antigens in common with clinically used purified protein derivative [PPD] preparation for skin testing patients for tuberculosis exposure. This classic delayed hypersensitivity test was applied to assess CMI in the chickens $(41,42)$. Before sacrifice, wattle thickness was measured with skinfold calipers and $0.2 \mathrm{ml}$ of PPD 250 tubercular units was injected intradermally. The site was remeasured at $48 \mathrm{~h}$ and the increment in thickness recorded. Normal, control, and GBM-immunized chickens were tested for wattle thickness increments to PPD.

Blood biochemical studies. The Technicon SMAC (Technicon Instruments Corp., Tarrytown, NY) was used to determine the serum creatinine, total serum protein, and serum albumin in samples obtained at sacrifice

Flow diagram. The sequence of studies and procedures in the first phase are summarized in the flow diagram below:

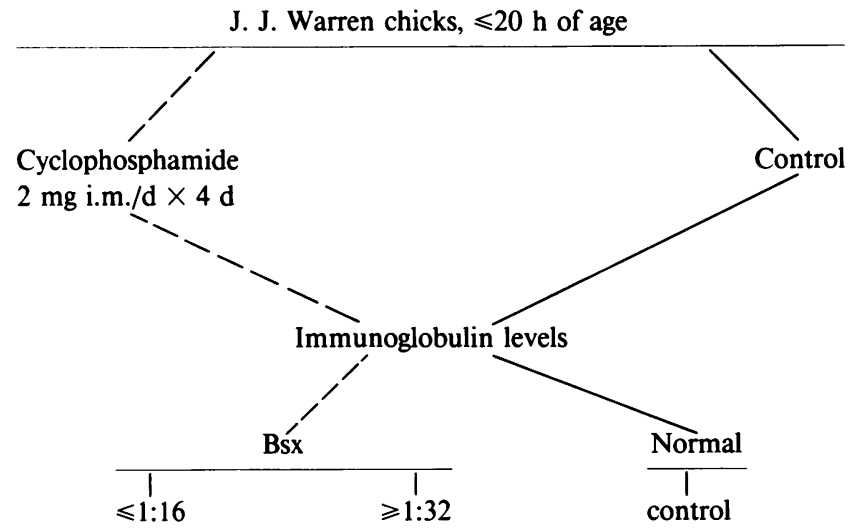

Week 5: IgG titers; week 6: SRBC immunization; week 7: Bleed for anti-SRBC titers; SRBC immunization; week 8: Bleed for anti-SRBC titers; select Bsx chickens with low IgG levels and low anti-SRBC response; week 9: Immunize with GBM or CFA, four groups: Bsx-CFA, BsxGBM, control-CFA, and control-GBM. Weeks after immunization (5, $7,9,11,13,15,17,19)$ : Wattle test; bleed and sacrifice for tissue and serum.

\section{Phase II}

CHARACTERIZATION AND QUANTITATION OF GBM DEPOSITS. a) Elution of antibody from control-GBM and Bsx-GBM animals. Kidneys from control chickens with documented in vivo deposits of IgG along the basement membrane were harvested, weighed, and minced in PBS at $4^{\circ} \mathrm{C}$. After homogenization for $30 \mathrm{~s}$ in a Waring microblender (Waring Products Div., Dynamics Corp. of America, New Hartford, $\mathrm{CT}$ ) in the cold, the tissue was repeatedly washed in PBS with centrifugation until the supernatant was clear. The sediment was eluted three times with three volumes of $0.02 \mathrm{M}$ citrate-citric acid saline buffer, $\mathrm{pH}$ 3.2 , for $1 / 2,1$, and $1 \mathrm{~h}$ at $37^{\circ} \mathrm{C}$. After centrifugation, the supernatants were brought to $\mathrm{pH} 7.3$ with sodium hydroxide and chilled, the supernatants were neutralized and combined, and the pooled eluate dialyzed against PBS for $48 \mathrm{~h}$ with frequent changes of buffer. The eluate was concentrated by precipitation with ammonium sulfate at $95 \%$ saturation, redissolved in water and precipitated with ammonium sulfate at $50 \%$ saturation, redissolved in PBS, and dialyzed again phosphate buffer. It was radiolabeled with $\mathrm{I}^{125}$ by the lactoperoxidase method (New England Nuclear, Boston, MA), dialyzed against $0.167 \mathrm{M}$ borate-buffered saline, $\mathrm{pH} 8.2$, ionic strength equals 0.16 , then slowly layered onto a $2.5 \times 55$ cm Sephadex G-200 column equilibrated with the same buffer and eluted at a flow rate of $11 \mathrm{ml} / \mathrm{h}, 4.8 \mathrm{ml} /$ tube. Repeated runs of normal chicken IgG (Cappel Laboratories Inc.) demonstrated reproducible elution of chicken IgG. The peaks of the column were examined by double 
diffusion in micro-Ouchterlony plates using anti-chicken IgG, IgA, and IgM. Specific activity was determined in duplicate from eluted labeled IgG using a single tube from the apex of the IgG peak which, after concentration, showed no other detectable proteins, and demonstrated a single line in double diffusion and immunoelectrophoresis against antichicken whole serum and anti-chicken IgG. The specific activity was $9.5 \times 10^{6} \mathrm{cpm} / \mathrm{mg}$ of $\mathrm{IgG}$. The presence of antibody to GBM was sought in column fractions by indirect immunofluorescence and verified to be present only in the IgG peak from the animals with in vivo deposits of chicken IgG. Kidneys of Bsx-GBM immunized chickens that had no detectable IgG present along the GBM by immunofluorescence were similarly pooled and eluted. The eluate was prepared in a fashion identical to that from GBM antibody-positive chickens, except it was not radiolabeled, but eluted and concentrated.

b) Calculations of kidney-fixing antibody (KFAb). Various quantities of chicken IgG anti-GBM antibody radiolabeled with $\mathrm{I}^{125}$ were injected intravenously into normal chickens. The animals were exsanguinated $2 \mathrm{~h}$ after injection. Samples of whole blood were counted and both kidneys were harvested, weighed, homogenized in a Waring blender (Waring Products Div.), and repeatedly washed in cold PBS until the supernatant contained minimal amounts of $\mathrm{I}^{125}$ and was clear. The entire washed homogenate from each pair of kidneys was counted in $1 \mathrm{ml}$ vol in a Beckman Gamma-8000 scintillation counter (Beckman Instruments, Inc., Fullerton, CA) with a 3-in. iodide crystal. Radioactivity was corrected for decay of $\mathrm{I}^{125}$ and background counts. Normal chicken IgG obtained from Cappel Laboratories Inc. was similarly chromatographed, labeled with $\mathrm{I}^{125}$ as described, and the specific activity adjusted to be the same as the injected anti-GBM antibody. Normal chickens received various quantities of the radiolabeled normal chicken IgG and the blood and kidneys were counted as described above. The amount of KFAb was determined from the cpm bound to kidney, and corrected for nonspecific binding using the specific activity of the IgG injected.

c) Determination of limits of detection of chicken IgG. Chicken IgG fractions of known KFAb concentration as determined above were injected into six chickens in six different doses. Animals were killed $1 \mathrm{~h}$ later and cryostat sections of kidneys were cut in triplicate and intermingled with each other, as well as with sections from normal and immunized chickens with in vivo GBM deposits of IgG of various intensity. Two sections were mounted on each slide and all were coded so that Dr. Bolton, of this institution, did not know whether the biopsy was from a normal chicken, a GBM recipient chicken kidney biopsy, or from an eluate recipient chicken. The intensity of fluorescence was then independently evaluated using the immunofluorescence grading scale previously detailed. This procedure was repeated on two separate additional days, again with intermingling of all the sections and reading in blind. The mean fluorescence intensity for each amount of KFAb injected was plotted on semilogarithmic paper to ascertain the limits of ability to detect GBM fixation of chicken IgG in vivo.

b) Determination of number of glomeruli in chicken kidneys. To determine the total number of glomeruli present per kidney in normal chickens, four chicken kidneys were individually homogenized in PBS and aliquots of well-suspended solution were counted in duplicate or triplicate from each kidney using phase-contrast microscopy. The results are presented as number of glomeruli/kidney or number per gram total kidney weight.

Attempts to transfer glomerulonephritis with nephritic eluate or sensitized cells

(a) INTRAVENOUS INJECTIONS OF ELUATE. Quantities of KFAb capable of inducing various degrees of in vivo staining after injection were administered intravenously to six normal chickens, two each at three dose levels, to produce a spectrum of in vivo GBM deposits of IgG. Chicken kidneys were biopsied at 14 and $30 \mathrm{~d}$, and animals sacrificed at $6 \mathrm{wk}$. Sections were evaluated by light and immunofluorescence microscopy. The sections read by immunofluorescence were coded in the fashion described above for determination of limits of detection of chicken IgG and read in blind with sections included from normal animals, nephritic GBM birds containing antibody on the basement membrane, and sections of specimens used in "c" above for determination of IgG deposits. Light microscopic sections were also read blindly for evidence of nephritis.

(b) ADMINISTRATION OF SENSITIZED CELlS. 1) Donor chickens. Additional J. J. Warren chicks 2-20-h old were placed in a filtered atmosphere room with sterilized water and Bsx with cyclophosphamide. Animals were evaluated for IgG in $1 \%$ agarose, and representative low and high responders were twice immunized with SRBC as described in the first part of the study. Birds were selected for immunization with GBM, which was first performed at 12 wk of age. There were 26 BsxGBM animals, 27 Bsx-CFA, 17 control-GBM, and 18 control-CFA birds in this portion of the study. $6 \mathrm{wk}$ after immunization, selected animals from each group were examined for circulating anti-GBM antibody by the indirect method. Animals used for transfer studies were exsanguinated, and suspensions were prepared from spleens by mincing and pressing sequentially through a 60-, 100-, 325-, and 400-gauge sieve, and then by passing suspensions through 23- and 25-gauge needles, followed by washing twice with RPMI 1640 . Spleen was selected because in vitro blast transformation studies of peripheral lymphocytes, spleen cells, and lymph node cells suggested that the best response was present in spleen cells (unpublished observation). Cell viability was assessed by trypan blue exclusion and was $>70 \%$. Normal unmanipulated 6-wk-old chickens received intravenous cells in a concentration from $4 \times 10^{7}$ to $1 \times 10^{9}$ per injection. Biopsies were taken from one kidney at 0,15 , and 30 min, and $4 \mathrm{~h}$ after infusion of cells, and at $24 \mathrm{~h}$ from the opposite kidney. The presence of any antibody along the GBM in immunized donor chickens was ascertained by immunofluorescence at the time of harvesting of cells. Recipient animals were killed 3 and $7 \mathrm{~d}$ later with light microscopic examination of kidney tissue.

2) Autoradiography. Other chickens received cells with three injections of tritiated thymidine $(1 \mu \mathrm{Ci} / \mathrm{g}$ body $\mathrm{wt}), 6.7 \mathrm{Ci} / \mathrm{mmol} \mathrm{sp}$ act (New England Nuclear). These injections of $\left[\mathrm{H}^{3}\right]$ thymidine were given at 4 , 19 , and $26 \mathrm{~h}$ after injection of cells. Animals were biopsied pre-, 1-3 d later, and sacrificed $1 \mathrm{wk}$ after cells. Autoradiographs were prepared from thick sections of plastic embedded tissues $3 \mu \mathrm{m}$ in thickness. Slides were dipped in nuclear track emulsion NTB-2 (autoradiographic supplies are from Eastman Kodak Co., Rochester, NY) and exposed for varying times. After development with D-19 and fixation with Ektaflo (Eastman Kodak Co.), sections were stained with H\&E and PAS reagent. Optimal time of exposure was determined by incubating slides for 7, 10,16, and $30 \mathrm{~d}$. The 16 and $30 \mathrm{~d}$ appeared similar, so 20 -d exposure times were used thereafter. The slides were examined in detail and all of the grains present within all glomeruli of each specimen were counted. Cells within capillary lumina were not counted, however.

Statistical analysis. Data are expressed as the mean \pm SEM. All data are analyzed by the $t$ and Chi square tests (43). Dilutions are presented as the reciprocal titer.

\section{Results}

\section{Effect of Bsx on immune response}

a) Chickens. Animals were successfully raised in the isolation environment with little morbidity or mortality. 70 of 75 Bsx 
Table I. Body Weights of Experimental Chickens (g)

\begin{tabular}{|c|c|c|c|}
\hline Group & $\mathbf{M}$ & $\mathbf{F}$ & $\mathbf{P}$ \\
\hline Bsx-GBM & $\begin{array}{l}3,169 \pm 92 \\
\text { (4) }\end{array}$ & $\begin{array}{l}2,109 \pm 102 \\
(12)\end{array}$ & $<0.001$ \\
\hline Bsx-CFA & $\begin{array}{l}3,116 \pm 76 \\
(7)\end{array}$ & $\begin{array}{l}2,105 \pm 98 \\
(9)\end{array}$ & $<0.001$ \\
\hline Bsx & $\begin{array}{l}3,302 \pm 159 \\
\text { (3) }\end{array}$ & $\begin{array}{l}2,063 \pm 106 \\
(5)\end{array}$ & $<0.001$ \\
\hline Nl-GBM & $\begin{array}{l}3,274 \pm 102 \\
(5)\end{array}$ & $\begin{array}{l}2,399 \pm 134 \\
(4)\end{array}$ & $<0.005$ \\
\hline Nl-CFA & $\begin{array}{l}3,519 \pm 105 \\
(6)\end{array}$ & $\begin{array}{l}2,236 \pm 48 \\
(5)\end{array}$ & $<0.001$ \\
\hline Bsx-all & $\begin{array}{l}3,171 \pm 56 \\
(14)\end{array}$ & $\begin{array}{l}2,099 \pm 59 \\
(26)\end{array}$ & $<0.001$ \\
\hline $\mathrm{Nl}$-all & $\begin{array}{l}3,408 \pm 80 \\
\text { (11) }\end{array}$ & $\begin{array}{l}2,045 \pm 264 \\
(9)\end{array}$ & $<0.001$ \\
\hline \multicolumn{3}{|c|}{ M-Bsx-GBM vs. nl-GBM } & NS \\
\hline \multicolumn{3}{|c|}{ M-Bsx-CFA vs. nl-CFA } & $<0.01$ \\
\hline \multicolumn{3}{|c|}{ F-Bsx-GBM vs. nl-GBM } & NS \\
\hline \multicolumn{3}{|c|}{ F-Bsx-CFA vs. nl-CFA } & NS \\
\hline
\end{tabular}

M, male; F, female; Bsx, cyclophosphamide bursectomized; NS, not significant. chickens were alive at 5 wk (93\%) compared with 25 of 25 control birds. During the course of the study, four additional Bsx animals died of inapparent causes, while there were no deaths in the control animals. Thus, overall survival in Bsx birds was $88 \%$ compared with $100 \%$ for normal animals. No difficulties were encountered with infections, poor oral intake, or malnutrition in any of the birds. However, to ascertain any effect Bsx might have on body weight, another group of 60 chickens of the same four study groups was weighed at the 26th wk of age (Table I). In these animals, body weight was less in male birds than in females in each study group, and male adjuvant birds were heavier than male Bsx-adjuvant birds. There were no differences between GBM-immunized birds of the same sex, whether normal or Bsx.

b) Bsx. Bursal tissue or remnants were weighed and examined histologically in chickens at the time of sacrifice. $13 \mathrm{wk}$ after immunization (22 wk of age) and later, little or no bursal tissue was present in any birds. Therefore, the first $13 \mathrm{wk}$ after immunization were used to compare Bsx and normal chickens' bursae. In Bsx birds, only remnant lymphoid follicles and disorganized architecture were found (Fig. 1). These findings varied in severity, but generally there was severe depletion of lymphoid cells compared with normal chickens. Bursal weights in the Bsx chickens were significantly less than normal controls, $P<0.0025$ (Table II). The same type of depletion and decreased bursal weights were observed at each sacrifice period, week 5-13.

c) Immunoglobulin levels. Serial titers of chicken IgG ranged from 32 to $>256$ in normal birds at 5-6 wk of age (mean $113.9 \pm 17.3, n=25$ ). Bsx bird IgG titers ranged from $<2$ to

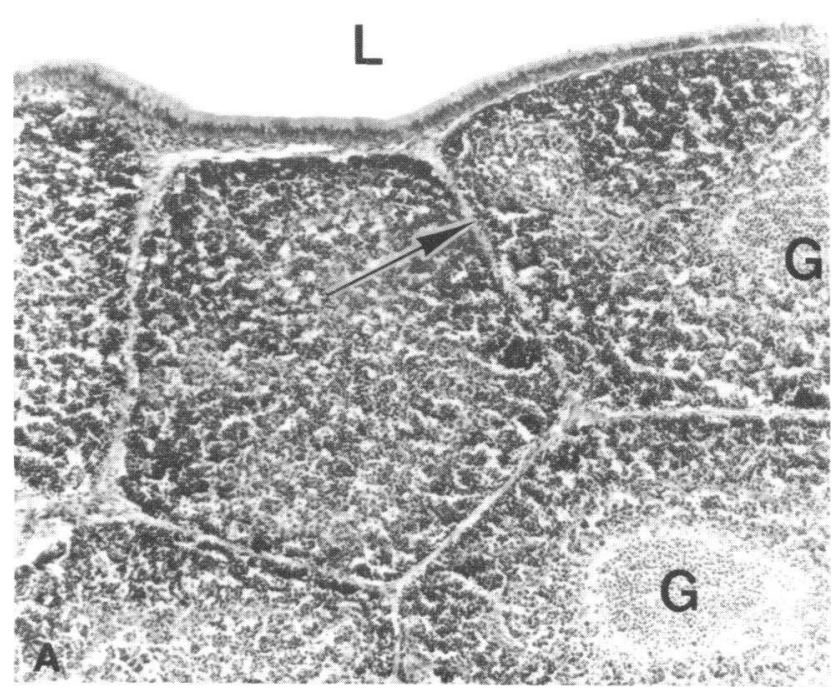

Figure 1. The effect of cyclophosphamide on the histology of the bursa of Fabricius. $(A)$ Normal morphology is illustrated. The lumen (L) of the bursa is lined by tall columnar epithelium stretched over a thin fibrovascular stroma (arrow) subdividing lymphoid follicles. Two follicles contain germinal centers $(G)$. The muscularis and adventitial components of the wall are not seen in this view. $(H \& E \times 60)$. $(B)$

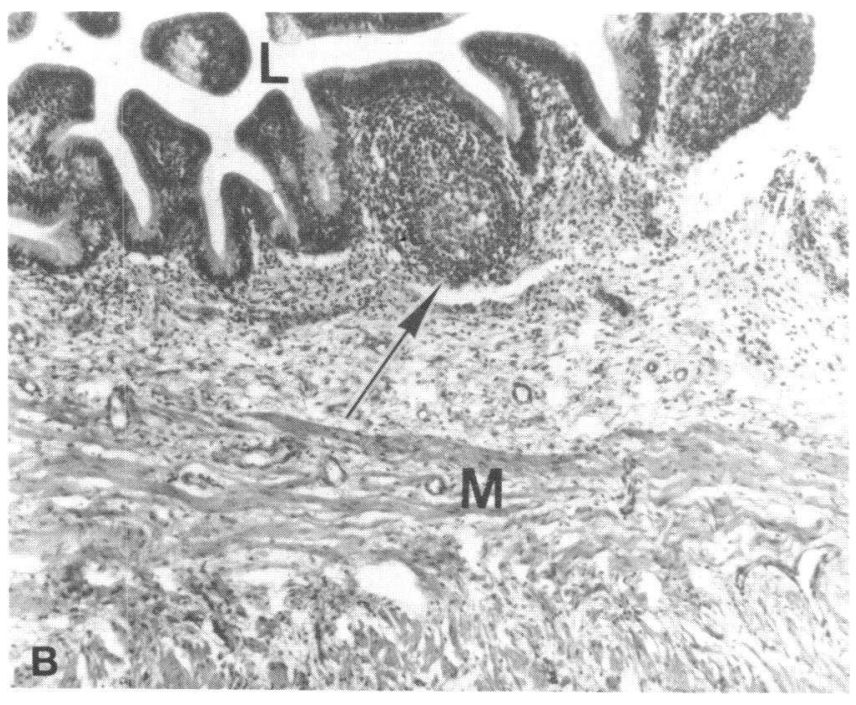

Bursa from an 18-wk-old chicken following neonatal Bsx with cyclophosphamide. The mucosa is folded and the submucosa shows marked attenuation of follicles (arrow) with complete absence of follicles in most fields. A mild, diffuse lymphocytic infiltrate is seen and the thickness of the bursa from the lumen $(\mathrm{L})$ to the muscularis $(\mathrm{M})$ is greatly reduced. (H\&E $\times 60$ ). 
256. $18 \%$ of Bsx animals had titers $64-256,25 \%$ a titer of 32 , and the remaining $56 \%$ had titers of $\leqslant 16$. The mean titers in birds selected for immunization was $14.2 \pm 1.6, n=43$ (Table II). This was significantly less than control birds, $P<0.0001$. Immunoelectrophoresis of sera from normal birds revealed strong IgG lines. Bsx birds with low titers of IgG had absence of the IgG line or only a trace remnant as measured by electrophoresis. IgM was present in all animals.

d) SRBC immunizations. We assessed the ability of Bsx and normal birds to mount a humoral immune response using SRBC. Bsx birds with low, medium, and normal IgG levels (titers $\leqslant 16,32-64$, and $>128$ ) were selected to receive SRBC as described in Methods. Four each of normal and medium responders, and 11 low responder birds were injected (Table II). Titers in normal chickens were $162 \pm 59$, which decreased to $33 \pm 12$ using $0.2 \mathrm{M}$ mercaptoethanol-treated serum. Titers in medium responders were $68 \pm 63$ for normal serum and $9 \pm 7$ in IgMinactivated serum. Two Bsx medium-IgG-level birds did not develop any antibody response. None of the low responder Bsx birds had any agglutinating activity against SRBC at 2 wk in either normal or mercaptoethanol-treated serum. Thus, Bsx chickens with low titer IgG as measured by immunodiffusion also failed to mount a humoral response to stimulation with the complex antigen SRBC.

e) Wattle thickness increments. The wattle thickness in all birds before injection of intracutaneous PPD was 2-3 mm in 48 birds. The thickness at $48 \mathrm{~h}$ after PPD ranged up to $11 \mathrm{~mm}$. The mean increment of Bsx-GBM birds was $3.89 \pm 0.45 \mathrm{~mm}$, compared with $3.37 \pm 0.75 \mathrm{~mm}$ in control-GBM chickens, which was not significantly different (Table II). An increase in wattle thickness developed in $93 \%$ of Bsx-GBM animals and $73 \%$ of control-GBM, $P=$ NS. 12 normal nonimmunized chickens had no detectable wattle thickness increment to PPD $48 \mathrm{~h}$ after skin tests were placed.

Table II. Immunologic Responsiveness in Control and Bsx Chickens

\begin{tabular}{|c|c|c|c|c|}
\hline & Bsx & Control & Bsx-GBM & Control-GBM \\
\hline $\begin{array}{l}\text { Bursal weight } \\
\qquad\left(g^{*}\right)\end{array}$ & $\begin{array}{l}0.38 \pm 0.11 \\
(4)\end{array}$ & - & $\begin{array}{l}0.89 \pm 0.23 \\
(19)\end{array}$ & $\begin{array}{l}2.92 \pm 0.91 \ddagger \\
(6)\end{array}$ \\
\hline $\begin{array}{l}\text { IgG levels, } \\
\text { titer }^{-1}\end{array}$ & $\begin{array}{l}14.2 \pm 1.6 \\
(43)\end{array}$ & $\begin{array}{l}113.9 \pm 17.3 \S \\
(25)\end{array}$ & - & - \\
\hline $\begin{array}{c}\text { Anti-SRBC } \\
\text { levels, } \\
\text { titer }^{-1}\end{array}$ & $\begin{array}{l}0 \pm 0 \\
(11)\end{array}$ & $\begin{array}{l}162.0 \pm 59.0 \ddagger \\
(4)\end{array}$ & - & - \\
\hline $\begin{array}{l}\text { Wattle } \\
\text { increment } \\
(\mathrm{mm})\end{array}$ & - & - & $\begin{array}{l}3.89 \pm 0.45 \\
(28)\end{array}$ & $\begin{array}{l}3.73 \pm 0.75 \\
(11)\end{array}$ \\
\hline
\end{tabular}

$n$ is given in parentheses.

* Through 13 wk.

$\ddagger P<0.0025$.

$\S P<0.0001$.
Table III. Histopathologic Parameters in Control and Bsx Chickens

\begin{tabular}{lllll}
\hline & Bsx & Control & Bsx-GBM & Control-GBM \\
\hline $\begin{array}{l}\text { Circulating } \\
\text { anti-GBM } \\
\text { titers }\end{array}$ & - & - & $<98.8 \pm 37.7^{-1}$ & $>2188 \pm 532 \ddagger$ \\
$\begin{array}{l}\text { IgG linear } \\
\text { deposits } \\
\text { along GBM, }\end{array}$ & - & - & $0.53 \pm 0.16 \S$ & $2.19 \pm 0.32^{\prime \prime}$ \\
$\begin{array}{l}\text { 0-4+I } \\
\text { Glomerular }\end{array}$ & $14.9 \pm 1.5$ & $13.6 \pm 0.8$ & $20.8 \pm 0.6$ & $(13)$ \\
size $(U)^{* *}$ & $(6)$ & $(3)$ & $(37)$ & $(13)$ \\
\hline
\end{tabular}

$n$ is given in parentheses.

* $68 \%$ totally negative by hemagglutination.

$\ddagger P<0.005$.

$\S 64 \%$ totally negative.

" $P<0.001$.

ף Limits of detection by fluorescence, $<6 \mathrm{fg}$ chicken IgG/glomerular section.

** Glomerular size: Bsx vs. Bsx-GBM, $P<0.001$; control vs. GBM, $P<0.05$; Bsx vs. control, $P=\mathrm{NS}$; control vs. Bsx-GBM, $P<0.005$; Bsx vs. GBM, $P<0.05$; GBM vs. Bsx-GBM, $P=$ NS.

\section{Development of experimental glomerulonephritis}

a) Anti-GBM antibody levels. All of the control-GBM birds developed circulating anti-GBM antibodies. Titers ranged from 256 to $>4,096$ with a mean of $>2,118 \pm 532$ (Table III). Only 12 of 37 (32\%) Bsx-GBM birds tested developed circulating anti-GBM antibody, which ranged from 2 to 512 (mean $<312 \pm 95, P<0.005$ ). The mean of the anti-GBM antibody for all Bsx-GBM birds combined was $99 \pm 38, P$ $<0.001$. Birds that had circulating anti-GBM antibody against bovine kidney sections were also positive against chicken kidney sections, but this was not quantitated. Positive sera were positive both by indirect immunofluorescence and hemagglutination assay.

b) Immunofluorescence findings on kidney tissue. At 5 wk after immunization, normal chickens had developed linear deposits of IgG along the GBM as previously described (30). During the ensuing weeks the intensity of deposits increased from $1+$ to $3-4+$ in all control birds. In contradistinction, GBM deposits were seldom observed in Bsx birds. $64 \%$ of Bsx chickens had no detectable IgG along the GBM. In the other $36 \%$ of animals, the intensity of linear staining was $<1.0+$ in half. Thus, $82 \%$ of animals developed $0-1+$ IgG deposits. Using the 11-point 0-4+ scale (38), the intensity of linear staining for IgG on the GBM in normal controls was $2.19 \pm 0.32$ compared with $0.53 \pm 0.16$ for Bsx-GBM, $P$ $<0.005$ (Table III). There were no deposits of C-3, other immunoglobulins, fibrinogen, or albumin in the animals. The Bsx-control and normal controls did not develop any deposits. 
c) Histopathology. Both Bsx-GBM and control-GBM chickens developed a proliferative glomerulonephritis associated with a marked increase in the mesangium, narrowing of capillary lumina, and diminution of Bowman's space (Fig. 2). Epithelial crescents were present in $24 \%$ of Bsx-GBM animals and $38 \%$ of control-GBM birds $(P=\mathrm{NS})$. The degree of crescent formation ranged from 2.1 to $23.5 \%$ in Bsx-GBM birds (14.3 $\pm 3.8 \%$ ), and from 2.3 to $25.0 \%$ in control-GBM animals $(15.2 \pm 2.3 \%), P=$ NS. Analysis of glomerular size (Table III) demonstrated that Bsx-GBM and control-GBM glomerular proliferation was similar, and both were significantly greater than either Bsx-control or normal-control chickens. There was no correlation between GBM deposits of IgG in Bsx chickens and proliferation, with birds having no detectable deposits developing equal degrees of glomerular proliferation and crescents.

The histologic changes in control and GBM-immunized animals are analyzed in a different fashion in Fig. 3. Sections were reread without knowledge of the previous qualitative or morphometric analysis or immunization status of the animal and assessed in terms of the percentage of glomeruli in a given section with proliferation from $0-4+$ and with crescents. 16-89 glomeruli were present per section (37.8 \pm 1.6$)$. A histologic index was then calculated by multiplying the percentage of glomeruli with a particular histologic grade by the grade, divided by 100 . Thus, the lowest score would be 0 and the highest possible score would be 4 . The histologic index is plotted against weeks after immunization and age in Fig. 3.

In the 5th wk after immunization, the killed GBM re-

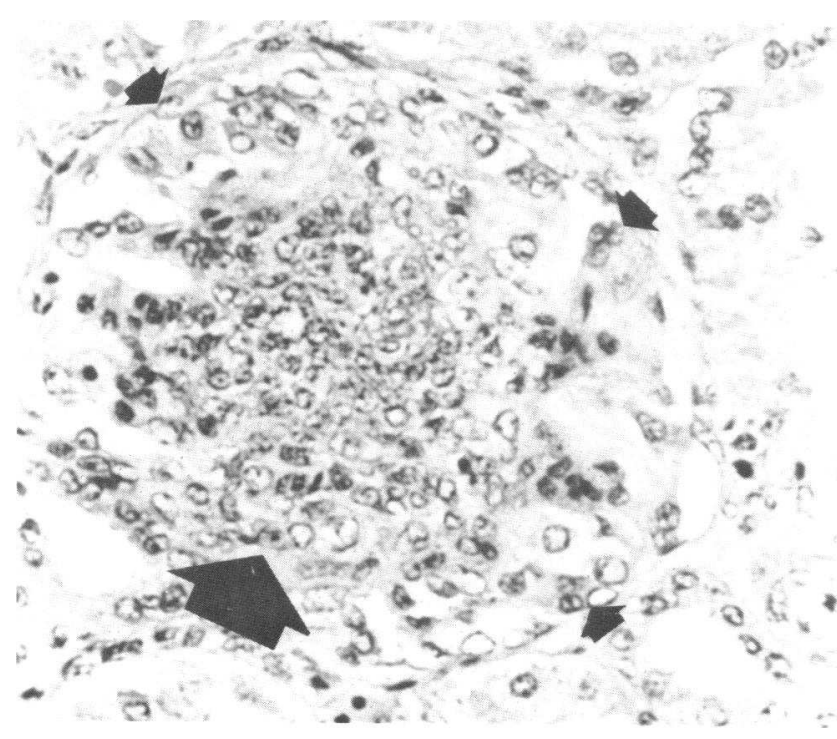

Figure 2. Glomerulus from an 18-wk-old Bsx chicken immunized with GBM. The mesangium is enlarged and hypercellular (large arrow) and a cellular crescent nearly surrounds the tuft (small arrows). Bowman's space is almost obliterated by the enlarged glomerulus and crescent. (H\&E $\times 450)$.

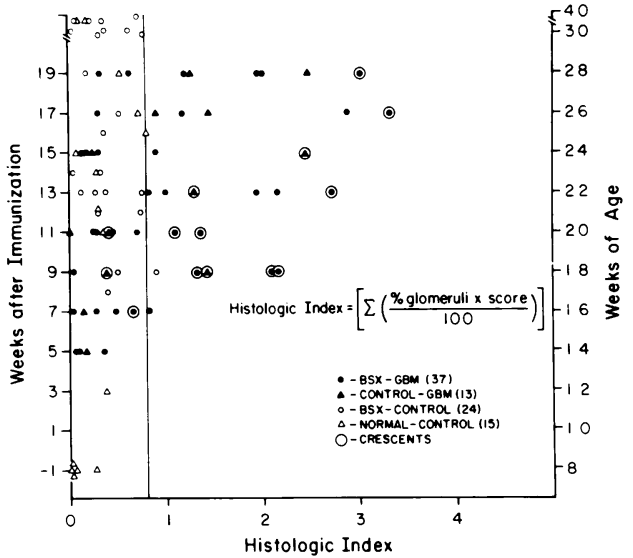

Figure 3. The percentage involvement and degree of glomerular proliferation (Histologic Index) and crescents are plotted relative to age of the animals and wk after initiation of immunizations. Bsx-GBM, control-GBM, Bsx-control, and normal controls are graphed. The vertical line at the Index of 0.8 is the upper limit for normal histologic findings.

cipient animals had histologic indices of 0.4 . By the 7th wk, the histologic index was a maximum of 0.82 . There was a gradual increase in the histologic index for the ensuing weeks, with animals in the 9th wk having a range of histologic indices to a high of 2.14. This trend continued throughout the remainder of the study with a shift of the histologic index to the right as a function of time. There was always a wide scatter of points, with some animals in each time period having minimal changes. The presence of crescents in each of the sections is also shown in Fig. 3, and was likewise associated with increase in length of time of immunization. The correlation coefficient for crescents relative to time after immunization in control-GBM animals was $r=0.73$, and for Bsx-GBM recipient animals was $r=0.81$. Fig. 3 illustrates that the histologic changes were somewhat more severe in Bsx-GBM than in control-GBM animals, since the symbols for Bsx-GBM recipient animals lie further to the right in each time period after immunization except for the 15 th wk.

Of 15 normal or CFA recipient animals and 24 BsxCFA or Bsx animals, some were followed to nearly $40 \mathrm{wk}$ of age, $>10$ wk longer than GBM recipient animals, to be certain that spontaneous aging changes did not account for the histologic lesions. Most of these animals had histologic scores of 0.6 or less, regardless of age, with only a few animals having scores greater than that, but all animals were $<1.0$. Furthermore, none of these animals had any crescents and they were all histologically distinct from animals immunized with GBM. 1 of 39 Bsx-CFA, CFA, and control animals had a histologic index of $\geqslant 0.8$, compared with 19 of $37 \mathrm{Bsx}$ GBM and 7 of 13 control-GBM animals. 8 of 37 Bsx-GBM and 2 of 13 control-GBM animals had histologic indices of $\geqslant 2.0$, and two animals, both in the Bsx-GBM group, had histologic indices $\geqslant 3.0$. Crescents were seen as early as $7 \mathrm{wk}$ 
Table IV. Association between Serum Anti-GBM

Levels and Time

\begin{tabular}{llllllllll}
\multicolumn{10}{c}{ Reciprocal of serum anti-GBM titers } \\
Weeks* & 0 & $\leqslant 8$ & 32 & 64 & 128 & 256 & 512 & 1,024 & $>4,000$ \\
\hline 5 & $(2)$ & $(1)$ & & & & $\ddagger$ & & & \\
7 & $(2)$ & $(3)$ & & & & $\ddagger$ & & & \\
9 & $(3)$ & & & & & $(1) \ddagger$ & & & $\ddagger$ \\
11 & $(2)$ & & $(1)$ & & $(2)$ & $(1)$ & $\ddagger$ & $\ddagger$ & \\
13 & $(3)$ & $(1)$ & & & & $(1)$ & & & $\ddagger$ \\
15 & $(1)$ & $(1)$ & & & $\ddagger$ & $(2)$ & & & $\ddagger$ \\
17 & $(2)$ & $(1)$ & & & & & $(1)$ & $\S$ \\
19 & $(4)$ & $(1)$ & & $(1)$ & & & $\ddagger$ & & $\ddagger$
\end{tabular}

$n$ of GBM-Bsx chickens is given in parentheses, total $n=36$.

* Weeks after first immunization.

¥ One GBM control chicken. $]$ Total $n=13$.
§ Two chickens.

after immunization in GBM recipient animals, even when the histologic index was low (0.4 and 0.6 , in three animals), but were more frequently present when the histologic index was $\geqslant 1.0$. Crescents were present in half of all animals with histologic index $\geqslant 1.0$. If the upper limits of a normal index is taken as 0.8 , as seems reasonable from Fig. 3, and crescents are considered as one form of nephritis, then 20 of 37 BsxGBM and 9 of 13 control-GBM chickens had disease, compared with 1 of 39 control animals with probable spontaneous nephritis, $P<0.001$.

d) Chronological and associative interrelationships among anti-GBM titers, GBM deposits of IgG, and histopathology. Tables IV-IX present data on the interrelationship between humoral antibody formation to GBM, in vivo deposits of $\mathrm{IgG}$ along the GBM, time, and histologic changes in glomeruli pre-

Table V. Time Course of Development of GBM Deposits of IgG by Immunofluorescence

\begin{tabular}{llllllllll}
\hline \multicolumn{7}{c}{ Immunofluorescence scores } \\
\cline { 2 - 8 } Week* $^{*}$ & 0 & Trace & $1+$ & $1-2+$ & $2+$ & $2-3+$ & $3+$ & $3-4+$ & $4+$ \\
\hline 5 & $(3)$ & & $\ddagger$ & & & & & & \\
7 & $(4)$ & $(1)$ & & & $\ddagger$ & & & \\
9 & $(3)$ & $\ddagger$ & & & & $(1) \ddagger$ & & \\
11 & $(3)$ & $(2)$ & & $\ddagger$ & $(1)$ & & & $\ddagger$ \\
13 & $(3)$ & $(1)$ & $(1)$ & & & & & $\ddagger$ \\
15 & $(2)$ & & & & & $(2) \ddagger$ & $\ddagger$ & \\
17 & $(1)$ & ND & & & $(1)$ & & $\ddagger$ & $(1) \ddagger$ \\
19 & $(6)$ & $\ddagger$ & & & $\ddagger$ & & &
\end{tabular}

$n$ of GBM-Bsx chickens is given in parentheses, total $n=36$. ND, not done. Frozen tissue was lost in one chicken.

* Weeks after first immunization.

$\ddagger$ GBM control chickens, total $n=13$.
Table VI. Correlation of Anti-GBM titers with GBM Immunofluoresence for IgG

\begin{tabular}{lrllllllll}
\hline \multirow{2}{*}{$\begin{array}{l}\text { Immuno- } \\
\text { fluorescence } \\
\text { on GBM }\end{array}$} & \multicolumn{7}{l}{ Reciprocal of serum anti-GBM titers } \\
\cline { 2 - 9 } & 0 & $\leqslant 8$ & 32 & 64 & 128 & 256 & 512 & 1,024 & $>4,000$ \\
\hline 0 & $(16)$ & $(6)$ & $(1)$ & $(1)$ & & $(1)$ & ND & & \\
$\operatorname{tr}$ & $(1)$ & $(1)$ & & & $(1)$ & $(1)^{*}$ & & & $*$ \\
$1+$ & $(1)$ & & & & & $*$ & & & \\
$1-2+$ & & & & & & & & $*$ & \\
$2+$ & $(1)$ & & & & $(1)$ & $*$ & $*$ & & \\
$2-3+$ & & & & & $*$ & $(3)$ & & & $*$ \\
$3+$ & & & & & & & & & $\ddagger$ \\
$3-4+$ & & $(1)$ & & & & & $*$ & & $\ddagger$ \\
$4+$ & & & & & & & & &
\end{tabular}

$n$ of GBM-Bsx birds is given in parentheses, total $n=36$. ND, not done. Frozen tissue was lost in one chicken.

* One GBM control bird.
$\ddagger$ Two birds.

sented both as qualitative morphologic changes and glomerular size. Accurate scoring of intensity of GBM deposits by fluorescence depends upon many factors: tissue background, observer experience, time of excitation, degree of protein fluoresceination, and others. These may especially affect a scoring of $0-1+$, such that discrimination between 0 -trace, trace, and trace-1+ may be difficult. In the tables, 23 birds totally negative and two birds negative to trace are listed as zero, while those with trace or trace- $1+$ are listed as trace. In Table IV, serum anti-GBM titers are compared with time after the first immunization. There was a trend toward a gradual increase in titer with time, with controlGBM animals in the 13-19th wk having, in many cases, titers

Table VII. Correlation between Histologic and Immunofluorescence Findings

\begin{tabular}{|c|c|c|c|c|c|c|c|c|c|c|}
\hline \multirow[b]{2}{*}{$\begin{array}{l}\text { IgG on } \\
\text { GBM }\end{array}$} & \multicolumn{10}{|c|}{ Light microscopic findings } \\
\hline & 0 & $\begin{array}{l}1+ \\
F\end{array}$ & $\begin{array}{l}1+ \\
D\end{array}$ & $\begin{array}{l}2+ \\
F\end{array}$ & $\begin{array}{l}2+ \\
\text { D }\end{array}$ & $\begin{array}{l}3+ \\
F\end{array}$ & $\begin{array}{l}3+ \\
\text { D }\end{array}$ & $\begin{array}{l}4+ \\
F\end{array}$ & $\begin{array}{l}4+ \\
D\end{array}$ & $\begin{array}{l}\text { Cres- } \\
\text { cents }\end{array}$ \\
\hline 0 & (2) & (8) & & (6) & (4) & & (4) & & (1) & (4) \\
\hline Trace & & $(2)^{*}$ & & (1) & & * & & (1) & & $(3)^{*}$ \\
\hline $1+$ & & $*$ & & (1) & & & & & & \\
\hline $1-2+$ & & * & & & & & & & & \\
\hline $2+$ & & * & & $(1)^{*}$ & & (1) & & & & \\
\hline $2-3+$ & & $(1)^{*}$ & & (1) & (1) & & * & & & * \\
\hline $3+$ & & & & $*$ & & & * & & & ${ }^{*}$ \\
\hline $3-4+$ & $*$ & & & & $\ddagger$ & & & & (1) & $(1)^{*}$ \\
\hline $4+$ & & & & ND & & & & & & \\
\hline
\end{tabular}

$n$ of GBM-Bsx chickens is given in parentheses, total $n=36$. F, focal; D, diffuse; ND, not done.

* One GBM control chicken. $]$ Total $n=13$.
$\ddagger$ Two chickens. 
Table VIII. Comparison of Glomerular Size to GBM Deposits of IgG

\begin{tabular}{|c|c|c|c|c|c|}
\hline \multirow{2}{*}{$\begin{array}{l}\operatorname{lgG} \\
\text { on GBM }\end{array}$} & \multicolumn{5}{|c|}{ Glomerular size (U) } \\
\hline & 12 & $13-17$ & $18-22$ & $23-27$ & 27 \\
\hline 0 & (1) & (6) & $(10)$ & (7) & (1) \\
\hline $\operatorname{tr}$ & & $*$ & $(2)^{*}$ & (1) & (1) \\
\hline $1+$ & & (1) & & $*$ & \\
\hline $1-2+$ & & & & & $*$ \\
\hline $2+$ & & & (1) $\ddagger$ & (1) & \\
\hline $2-3+$ & & & $(1) \ddagger$ & (2) & \\
\hline $3+$ & & $\S$ & $\ddagger$ & & \\
\hline $3-4+$ & & & (1) & & ND \\
\hline
\end{tabular}

$n$ of GBM-Bsx chickens is given in parentheses, total $n=36$. ND,

not done. Frozen tissue was lost in one chicken.

* One GBM control chicken.

$\ddagger$ Two chickens.

$\S$ Three chickens.

Total $n=13$.

$>4,000$. However, high titers were present in some animals as early as the 9 th wk, and titers of only 512 were recorded in animals as late as the 19th wk. Regression analysis did not show any statistical significance between anti-GBM levels and time. Bsx animals had little circulating antibody at any time during the study, including to the 19th wk. Animals with antibody present in 1:2 and 1:4 dilutions are included in the $\leqslant 8$ antiGBM titer column. Although a few Bsx-animals did have antibody titers of 256 and 512, most did not develop circulating antibodies or had only very little.

The time course of development of IgG along the GBM is presented in Table V. Control-GBM immunized animals had deposits by the 5 th wk, with a gradual tendency for the deposits to increase with time up to the 19th wk. However, there were animals with minimal deposits even in the 19th wk, and linear regression analysis of the time course relative to intensity of deposits was not significant. Bsx-GBM chickens usually developed no antibodies along the GBM, even by the 19th wk, and animals with 0-trace deposits comprised the majority of the group. A few animals had heavier deposits, but there was not an increasing number of animals or amount of deposits with time in Bsx chickens.

The relationship between anti-GBM titers and basement membrane deposits of $\mathrm{IgG}$ is presented in Table VI. There was a poor correlation between the titers and GBM deposits in the control-GBM animals, $r=0.49 .24$ of the Bsx-GBM chickens had GBM deposits of 0 -trace intensity and antibody titers of 0 to $\leqslant 1: 8$. There was no correlation between circulating antibody levels and intensity of IgG on the GBM in those few birds that did have positive sera for antibody and deposits along the GBM.

Table VII presents the data on correlation between the histologic and immunofluorescence findings. Sections were graded qualitatively in terms of whether the lesions were focal or diffuse, -with an intensity grade from $0-4+$. There was no correlation in control-GBM animals between the amount of IgG on the GBM and the associated histologic findings, with some animals having 3-4+ deposits of $\mathrm{IgG}$ and minimal light microscopic changes, while others had heavy deposits of $\operatorname{IgG}$ and severe histologic changes. Bsx-GBM recipient animals had a wide spectrum of histologic abnormalities, but the severity of histologic changes was not related to the amount of GBM deposits of IgG. Animals with 0 -trace deposits had histologic changes as severe as $4+$ diffuse proliferation with crescents. Seven of the nine Bsx-GBM animals with crescents had 0-trace deposits of $\mathrm{IgG}$ on the GBM.

Glomerular size is compared with GBM deposits in Table VIII. Normal glomerular size is $\leqslant 13 \mathrm{U}$. There was no correlation between intensity of GBM deposits of $\mathrm{IgG}$ and the size of the glomeruli in the control-GBM chickens, nor was there any correlation between size and deposits of Bsx animals. Similarly, there was no correlation between histologic abnormalities and serum anti-GBM titers in either the Bsx-GBM or control-GBM animals (Table IX).

e) Characterization and quantitation of GBM deposits. The KFAb of eluted nephritic kidneys was $0.8 \%$ of the total IgG eluted from the kidneys. The relationship between the $\mu \mathrm{g}$ of $\mathrm{KFAb}$ deposited per gram of wet weight in normal chickens and immunofluorescence staining intensity grade are presented in Fig. 4. Slightly $>2+$ linear deposits of chicken IgG result from the deposition of $<1 \mu \mathrm{g}$ of $\mathrm{KFAb} / \mathrm{g}$ of wet weight, and there was a linear relationship between the logarithm of the amount of KFAb bound and the immunofluorescence intensity. A small amount of KFAb resulted in trace amounts of staining that were similar to the intensity of GBM staining found in normal chickens. There was a very good correlation between the amount of KHAb per gram of wet weight in the injected

Table IX. Correlation between Histology and Serum Anti-GBM Titers

\begin{tabular}{|c|c|c|c|c|c|c|c|c|c|c|}
\hline \multirow[b]{2}{*}{$\begin{array}{l}\text { Reciprocal } \\
\text { titer }\end{array}$} & \multicolumn{10}{|c|}{ Histologic finding by light microscopy } \\
\hline & 0 & $\begin{array}{l}1+ \\
\mathrm{F}\end{array}$ & $\begin{array}{l}1+ \\
D\end{array}$ & $\begin{array}{l}2+ \\
F\end{array}$ & $\begin{array}{l}2+ \\
\mathrm{D}\end{array}$ & $\begin{array}{l}3+ \\
F\end{array}$ & $\begin{array}{l}3+ \\
\mathrm{D}\end{array}$ & $\begin{array}{l}4+ \\
F\end{array}$ & $\begin{array}{l}4+ \\
D\end{array}$ & $\begin{array}{l}\text { Cres- } \\
\text { cents }\end{array}$ \\
\hline 0 & $(2)$ & (6) & & (4) & (4) & (1) & $(2)$ & & & (3) \\
\hline$\leqslant 8$ & & (2) & & (3) & & & (1) & & $(2)$ & (2) \\
\hline 32 & & & & & & & (1) & & & (1) \\
\hline 64 & & (1) & & & & & & & & \\
\hline 128 & & $(1)^{*}$ & & (1) & & & & & & \\
\hline 256 & & $(1)^{* * *}$ & & (2) & (1) & & & (1) & & $(3)^{*}$ \\
\hline 512 & * & & & $(1)^{*}$ & & & & & & \\
\hline 1,024 & & $*$ & & & & & & & & * \\
\hline$>4,000$ & & & & $*$ & $* *$ & * & ** & & & $* * *$ \\
\hline
\end{tabular}

$n$ of GBM-Bsx birds is given in parentheses, total $n=37$. F, focal; D, diffuse.

* One GBM control bird.

$\ddagger$ Two birds.

$\S$ Three birds.
Total $n=13$ 


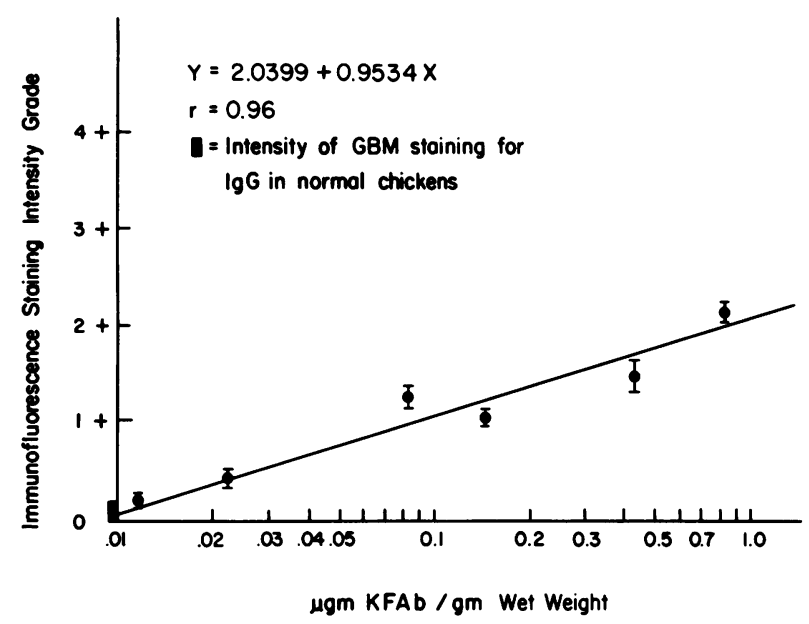

Figure 4. Immunofluorescent staining intensity for chicken IgG along the GBM is closely correlated with the amount of KFAb bound to

kidney. (Staining intensity $=2.0399+0.9534\left[\log \frac{\mu \mathrm{g} \mathrm{KFAb}}{\mathrm{g} \text { wet wt }}\right]$, $r=0.96, P<0.001)$. Lower limits of detection are $<6 \mathrm{fg} /$ glomerularsection.

kidneys and the immunofluorescence staining intensity, $r=0.96, P<0.001$. Note that the chickens used were all normal and thus had some background staining for IgG. In Bsx chickens there is essentially no IgG background and so the amount of detectable deposits of IgG would be considerably less than the $0.01 \mu \mathrm{g} / \mathrm{g}$ wet wt. There were $1.42 \pm 0.04$ million glomeruli in two kidneys, 710,000 glomeruli/kidney, or 164,352 glomeruli/ $\mathrm{g}$ of whole kidney. Since all of the IgG immunofluorescence was in glomeruli, the most accurate representation of immunofluorescence deposits of KFAb would be on the basis of IgG/ glomerulus. The average glomerulus is $\sim \mathbf{4 0}$ micra thick and cryostat sections are four micra in thickness. Thus, there would be $\sim 10$ sections/glomerulus and the amount of KFAb detected per glomerulus would be described by: KFAb/glomerulus $=(\mathrm{KFAb} \mu \mathrm{g} / \mathrm{g} \mathrm{wt}) /(164,352$ glomeruli $/ \mathrm{g} \times 10$ glomerular-sections/glomerulus $)=(\mathrm{KFAb}) \times\left(6.08 \times 10^{-7}\right) \mu \mathrm{g} /$ glomerularsection. Thus, using 4-micra thick sections, the amount of chicken IgG that was detected on GBM as shown in Fig. 4 was $6.08 \times 10^{-13} \mathrm{~g} /$ glomerular-section for a $2+$ fluorescence-staining intensity. Trace staining with slightly $>0.01 \mu \mathrm{g}$ of $\mathrm{KFAb} / \mathrm{g}$ of wet weight would represent $6.08 \times 10^{-15} \mathrm{~g} /$ glomerular-section or $\sim 6 \mathrm{fg}$ of chicken IgG/glomerulus in 4-micra thick sections. Therefore, in Bsx chickens with negligible IgG tissue background, several magnitudes $<6 \mathrm{fg} /$ glomerular-section would be readily detectable.

f) Nonspecific esterase. All kidney biopsies demonstrated heavy staining of esterase-positive tubular cytoplasm. In addition, intraluminal macrophages had darkly staining cytoplasmic granules. Chicken glomeruli contained mesangial cells that stained lightly in small granules (Fig. $5 A$ ). The staining was present in all chickens. Birds with proliferative nephritis developed an increased number of these faintly esterase-positive cells (Fig. $5 \mathrm{~B}$ ), but there were never any glomerular infiltrates of cells with heavy dark granules characteristic of macrophages. Esterase-positive cells were also not present within epithelial crescents or the interstitium. However, we did observe heavily esterase-positive cells within Bowman's space in close proximity to glomerular tufts and crescents (Fig. $5 C$ ).

g) Biochemical studies. Values from serum samples collected at sacrifice at each time interval were compared, and since no difference at time intervals were observed, these are considered together. The serum creatinines ranged from 0.2 to $0.5 \mathrm{mg} / \mathrm{dl}$ for Bsx-GBM, and from 0.2 to $0.8 \mathrm{mg} / \mathrm{dl}$ for control-GBM (mean, $0.35 \pm 0.01, n=36$ for Bsx-GBM vs. $0.38 \pm 0.05 \mathrm{mg} / \mathrm{dl}$, $n=12$ for controls, $P=\mathrm{NS}$ ). Serum albumin levels were lower in Bsx-GBM birds than in control-GBM, 1.74 $\pm 0.05(n=36)$ $\mathrm{g} / \mathrm{dl}$ Bsx compared with $1.96 \pm 0.08 \mathrm{~g} / \mathrm{dl}(n=12)$, controls; $P$ $<0.05$. Total protein values were also lower in Bsx-GBM birds, $3.3 \pm 0.13 \mathrm{~g} / \mathrm{dl}(n=36)$ compared with $4.3 \pm 0.21 \mathrm{~g} / \mathrm{dl}, n=12$, for control-GBM birds, $P<0.001$. Bsx and control animals not immunized with GBM had biochemical values comparable to their immunized cohorts.

\section{Attempts to transfer glomerulonephritis with nephritic eluate or sensitized cells}

a) Intravenous injections of eluates. Two chickens were both injected with $0.48,1.90$, and $4.76 \mu \mathrm{g}$ KFAb intravenously. These quantities of KFAb produced GBM staining of chicken IgG of intensities $1+, 1.5+$, and $2+$. Kidney tissue obtained from each of the six animals at 14 and $30 \mathrm{~d}$ by biopsy and at sacrifice at $6 \mathrm{wk}$ were examined without knowledge of dose of KFAb administered or the time after administration of KFAb. In none of the 18 tissue specimens were there any histologic abnormalities suggesting even a mild response to the injected antibody. Fluorescence deposits of the intensity anticipated were present in the 14- and 30-d biopsies with some diminution at $6 \mathrm{wk}$. The eluate from Bsx-GBM animals without deposits by immunofluorescence also contained no detectable $\mathrm{IgG}$ and no protein in the IgG elution area when run upon the Sephadex column. Thus, the solution from the IgG area, which was only buffer, was not injected into other animals.

b) Administration of sensitized cells. Eight normal chickens received either control or sensitized cells without $\left[\mathrm{H}^{3}\right]$ thymidine. No histologic changes were detectable in any of the birds. Six other normal animals received cells with $\left[\mathrm{H}^{3}\right]$ thymidine. Three each received cells harvested from donors immunized either with CFA or CFA and GBM. There were no histologic abnormalities in these birds, either. Grain counts were $1.6 \pm 1.1 / \mathrm{glo}-$ merulus in four of the six animals that had biopsies before infusion of cells. Biopsies were then obtained at 1-3 d, and 1 wk after the infusion of cells as described in Methods. The number of biopsy specimens obtained for the three recipients of normal cells were $n=6$, and none of the values were significantly different from each other. The mean of these was $4.2 \pm 3.2$, not significantly different from the preinfusion values of 1.6 grains/glomerulus. In contradistinction, in four biopsy specimens obtained from normal animals that were recipients of cells from Bsx-GBM immunized donors, cell counts were 

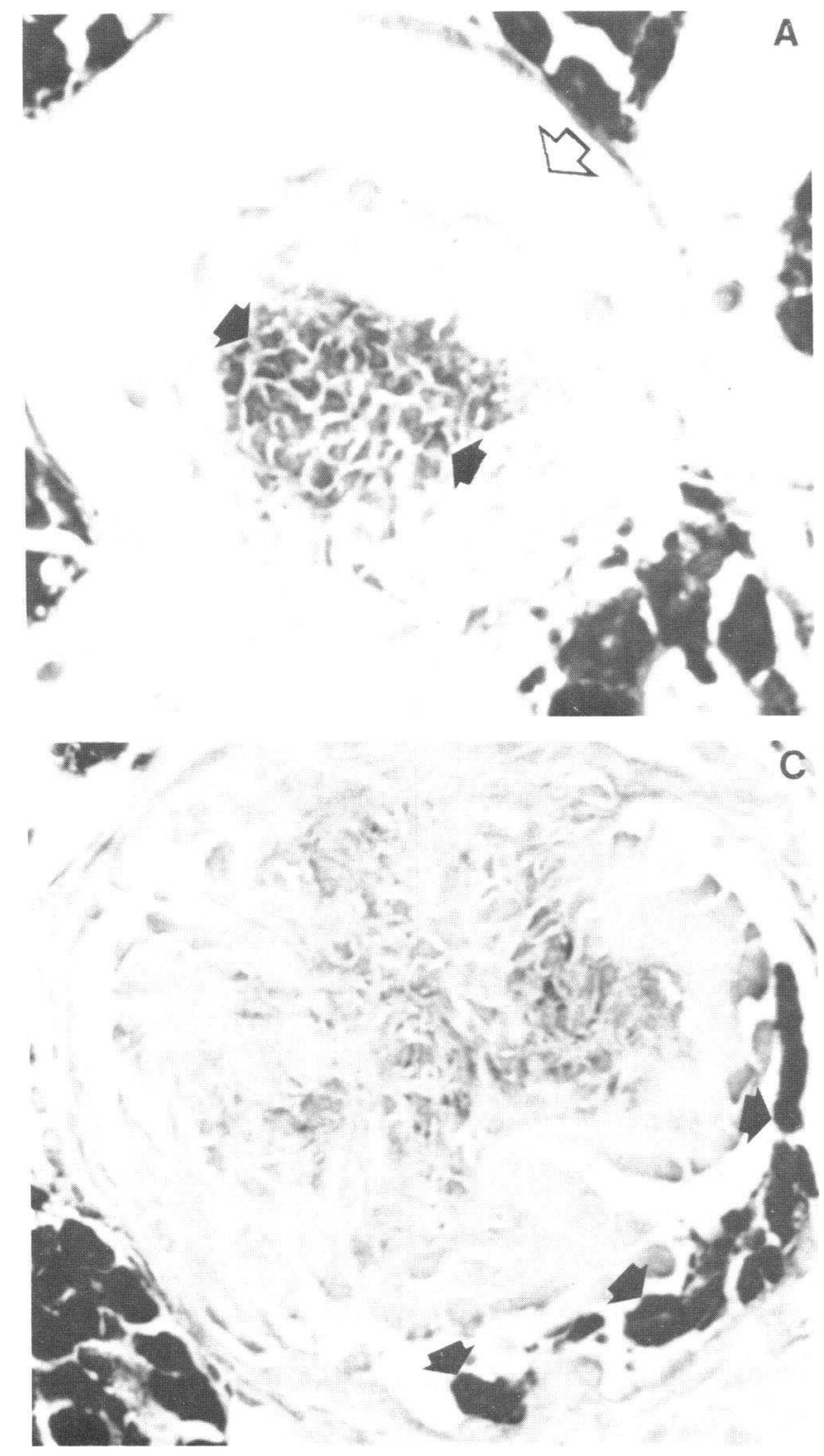

$18.2 \pm 11.8$ /glomerulus, significantly different from both the preinfusion values and from the CFA cell recipient chickens, $P<0.05$. All of the labeled cells were within the glomerular tuft within the mesangium. No grains in cells in the capillary lumina were counted.

\section{Discussion}

We have previously shown that functional $T$ cells are required to develop anti-GBM disease in mice $(28,29)$. Immunization of normal mice with human GBM predictably resulted in the development of antibody along the GBM. Immunization of $T$ cell deficient homozygous (nu/nu) mice did not elicit antibodies, while immunization of heterozygous hairy litter mates $(\mathrm{nu} /+)$

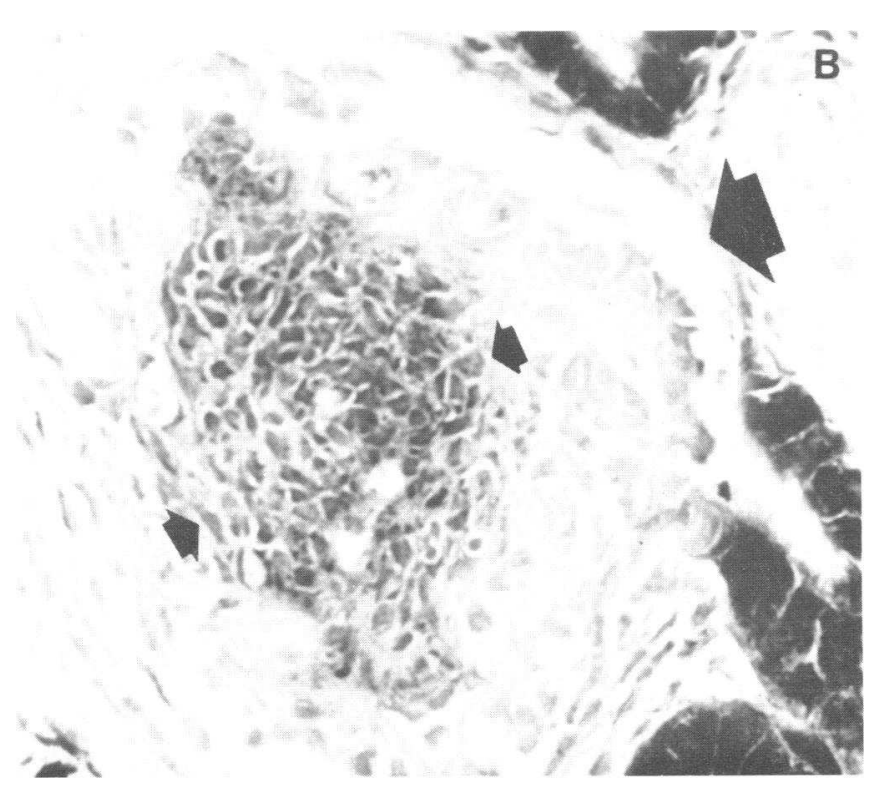

Figure 5. Coronal sections through chicken glomeruli stained for non-specific esterase. $(A)$ 28-wk-old normal chicken. Proximal tubular cells stain darkly, while mesangial cells stain with much less intensity (filled arrows). The tuft is normocellular with a characteristically large Bowman's space (empty arrow). $(\times 450)$. (B) An 18-wk-old BsxGBM chicken. The magnification is identical to Fig. $5 A$. There are many more esterase-positive cells in the mesangium, resulting in expansion and enlargement of the tuft (small arrows). Bowman's space is compromised (large arrow) by the tuft. $(\times 450)$. (C) A 26-wk-old Bsx-GBM immunized animal. Proximal tubular cells outside of Bowman's capsule stain heavily for esterase, as do numerous cells in Bowman's space in close approximation to the tuft (arrows). Intrinsic mesangial cells stain lightly for esterase. $(\times 450)$.

caused disease. This model established the absolute necessity of functional $\mathrm{T}$ cells for Steblay nephritis, but did not answer the question of the role of CMI without the humoral system. All previous models studying CMI in glomerulonephritis have used a planted antigen and relied on nephritis of "humoral" origin with recruitment of cellular components of the immune system $(8,12-15,17,18,44,45)$. To examine the individual roles of humoral and CMI we investigated the development of nephritis in a chicken model, which allowed us to virtually eliminate humoral immune system participation. We successfully produced humorally immunodeficient chickens by Bsx with cyclophosphamide. This was evidenced by bursal damage histologically, very low bursal weights, and inability to mount a humoral immune response. Nonetheless, they developed glo- 
merulonephritis, as previously described in normal chickens (30). The histologic severity of nephritis increased with time and number of immunizations. However, we were unable to show a correlation between length of immunization and development of anti-GBM antibodies, or intensity of GBM deposits of IgG in vivo. These findings suggest that the poor response of Bsx birds to SRBC was predictive of lack of response to GBM, despite repeated immunization, and that sluggishness of immune response was not the basis for absence of anti-GBM antibodies. It is probable that the degree of humoral impairment reflected the relative completeness of Bsx, such that some less impaired birds mounted a variable response, as shown by low titer GBM deposits and circulating antibody, while most were significantly depressed, and did not develop antibodies.

There was similarly no correlation between circulating antiGBM antibody or GBM deposits of IgG and the histologic lesion. Many birds with minimal or no deposits had lesions as severe or more so than those with antibody on the GBM. This lack of correlation was shown by several approaches, including quantitative measurements through coronal glomerular sections and examination of the distribution and severity of lesions in individual birds (Tables VII-IX). Further, the abnormalities were clearly not related either to cyclophosphamide, CFA, or the aging process (Fig. 3).

Development of nephritis, therefore, was a result of immunization with GBM. The ensuing nephritis could theoretically arise because of auto-antibody to GBM, a CMI response to GBM, humoral combined with CMI, or unexplained mechanisms. The amount of antibody bound to GBM in vivo in control-GBM birds was less than that which has been shown necessary to induce disease in mammals. Unanue and Dixon (46) showed that 150-200 $\mu \mathrm{g}$ ( $2.9 \mu \mathrm{g} /$ glomerulus) of heterologous $\mathrm{KFAb}$ in rats was required to induce disease, while Lerner and Dixon (47) demonstrated that deposited heterologous KFAb in the kidney produced proteinuria when $\geqslant 5 \mu \mathrm{g} / \mathrm{g}$ kidney weight was present. More homologous antibody was required. The quantities present in our avian model are less. Elution of nephritic kidneys yielded $46 \mu \mathrm{g} / 119 \mathrm{~g}$ whole kidney, or 0.39 $\mu \mathrm{g} / \mathrm{g}$. While we certainly lost some KFAb in the elution process, it is obvious from Fig. 4 and Tables VII and VIII that KFAb in vivo in control-GBM birds in the range of $0.01-1.0 \mu \mathrm{g} / \mathrm{g}$ weight, or $<6 \mathrm{pg} /$ glomerulus, were "associated" with nephritis. Thus, chickens might require less KFAb than mammals to produce disease, or the antibody may have little pathologic role. It is possible that nephritogenic quantities of $\mathrm{IgG}$ were present in those animals in which fluorescence was negative. This is unlikely, inasmuch as (a) our ability to detect bound IgG is $<6$ $\mathrm{fg} /$ glomerular-section and $<0.01 \mu \mathrm{g} / \mathrm{g}$ tissue; $(b)$ concentrated eluates of negative Bsx-GBM kidney contained no IgG by column chromatography and no anti-GBM activity by indirect immunofluorescence; and $(c)$ injection of KFAb into naive chickens in quantities far greater than our ability to detect the resultant GBM deposits produced no nephritis.

Thus, lack of correlation between deposits and nephritis and the presence of severe nephritis in the absence of documented humoral immune response, strongly bespeak pathogenetic mechanisms other than antibody-mediated nephritis. The Bsx animals developed cellular sensitization evidenced by wattle thickness increments to PPD after immunization with $M$. $t u$ berculosis H37Ra. Since a humoral immune response was absent by various parameters, the nephritis must, by exclusion, have arisen from participation of the cellular system, or by yet other mechanisms. In view of the possibility that this was indeed a CMI nephritis, we attempted to induce the disease by injection of cells from sensitized animals into naive recipients. Evidence for sensitization of these cells was indirect, since they came from nephritic animals, but were not shown directly to be sensitized. Compared with control cells from animals not immunized with GBM, there were increased mesangial grain counts, even though we could not show proliferation. These findings suggest that the infused cells were associated with uptake of label within the mesangium. Many weeks are required to develop nephritis in normal birds immunized with GBM, so it does not seem unreasonable that some greater length of time may be required to produce nephritis in naive recipients by transferred sensitized cells. Our cell transfer experiments were designed to be as short lived as possible and obviate the graft vs. host reaction that occurs with foreign lymphocyte injection. Thus, although transfers were between histoincompatible animals, we believe a graft vs. host reaction was unlikely to account for the increased grain counts in this short period of time. Use of syngeneic animals should permit cell transfer studies between animals that were not possible to perform here because of their random bred background. These studies are currently in progress and should allow serial long term investigations with cell transfers, including subspeciation of transferred cells.

If $\mathrm{CMI}$ is mainly responsible for the development of this nephritis, we do not know which components of that system may be involved. Faintly esterase-positive cells were present in the mesangium of normal chickens and the number of cells increased as the mesangium enlarged. The cells never assumed the appearance of macrophages of other species, nor of monocytes stained in the intravascular spaces in the chicken kidney sections. However, the present study does not eliminate a role for monocytes. Macrophages are capable of stimulating endothelial and other cellular proliferation (48). Increasing evidence implicates an important role for macrophages in the proliferative changes of nephritis $(8,13,18,45,49-53)$, and macrophage products appear to be intimately involved with glomerular injury.

However, since the cells comprising proliferative nephritis in our model did not appear to be lymphocytes or macrophages, we postulate that mesangial cell proliferation accounted for the increase in glomerular size. Hyperplasia of intrinsic mesangial cells causes glomerular proliferation in male kidney transplant recipients of female kidneys (54). Thus, some forms of mesangial growth may result from stimulation by lymphocyte or macrophage mediators. Alternatively, some type of intrinsic sensitization of mesangium resulting in proliferation may occur.

The glomerulonephritis in Bsx-GBM and control-GBM birds was quite severe. Nonetheless, no decrease in glomerular filtering ability was observed. Maintenance of normal filtration may be related to the unique structural-functional association of the 
glomerular capillary loops and the mesangium. Avian glomeruli are much smaller and more oriented to a central mesangium. Even with massive enlargement of the mesangium, the capillary loops simply remain on the periphery, surrounding the mesangium without compromising luminal cross sectional space to a significant degree. In addition, chicken leukocytes are deficient in proteolytic enzymes capable of injuring basement membrane and allowing protein leakage (55). Total serum protein levels were reduced in Bsx-GBM birds, probably related to the low IgG levels. The reason for the mild lowering of albumin is unclear, but may reflect some effect of cyclophosphamide on protein metabolism. Although we did not weigh the birds in phase I studies, similar groups of animals demonstrated weight differences only between males and females, suggesting that nutritional factors did not account for the differences in serum albumin.

In conclusion, we have immunized normal and Bsx chickens and produced anti-GBM nephritis in normal birds. Bsx chickens with documented severe depression of humoral immunity and intact CMI were unable to develop antibodies against the immunogen or their own GBM. Nonetheless, the Bsx chickens also developed a proliferative nephritis with crescents comparable to GBM-immunized controls. This nephritis developed in the absence of glomerular immunoglobulin, complement, or fibrinogen deposition, was not transferrable with nephritic eluates, but may potentially be transferrable by sensitized cells. Although CMI and humorally mediated immunity were not cleanly separated in all animals, the clear development of nephritis in those birds without apparent deposits strongly suggests, but does not prove, that CMI caused the lesion. These findings indicate that CMI may play a major role, albeit by unknown mechanisms, in the pathogenesis of this model of avian nephritis. By implication, CMI may be important in the pathogenesis of other kinds of nephritis as well.

\section{Acknowledgments}

We are grateful for the technical help of M. Santulli, D. Shifflett, T. Tyson, and P. Kirkpatrick, and to Dr. Donald Keefer for the autoradiographic studies. The secretarial assistance of B. Rogers is gratefully acknowledged. This work was supported in part by U. S. Public Health Service grants AM 21484 and AM 32530.

\section{References}

1. Dixon, F. J., and C. B. Wilson. 1979. Immunological renal injury produced by formation and deposition of immune complexes. In Immunological Mechanisms of Renal Disease. C. B. Wilson, B. M. Brenner, and J. H. Stein, editors. Churchill Livingston, Inc., New York. 1-34.

2. Wilson, C. B., and F. J. Dixon. 1979. Renal injury from immune reactions involving antigens in or of the kidney. In Immunological Mechanisms of Renal Disease. C. B. Wilson, B. M. Brenner, and J. H. Stein, editors. Churchill Livingston, Inc., New York. 35-66.

3. Dixon, F. J. 1970. What are sensitized cells doing in glomerulonephritis? New Engl. J. Med. 283:536-537.

4. Min, K. W., F. Györkey, P. Györkey, J. J. Yium, and G. Eknoyan. 1974. The morphogenesis of glomerular crescents in rapidly progressive glomerulonephritis. Kidney Int. 5:47-56.
5. Atkins, R. C., S. R. Holdsworth, E. F. Glasgow, and F. E. Matthews. 1976. The macrophage in human rapidly progressive glomerulonephritis. Lancet. I:830-832.

6. Morita, T., Y. Suzuki, and J. Churg. 1973. Structure and development of the glomerular crescent. Am. J. Pathol. 72:349-359.

7. Monga, G., G. Mazzucco, G. B. di Belgiojoso, and G. Busnach. 1979. The presence of possible role of monocyte infiltration in human chronic proliferative glomerulonephritis. Am. J. Pathol. 94:271-284.

8. Schreiner, G., R. Cotran, V. Pardo, and E. Unanue. 1978. A mononuclear cell component in experimental immunological glomerulonephritis. J. Exp. Med. 147:369-384.

9. Atkins, R. C., E. F. Glasgow, S. R. Holdsworth, N. M. Thomson, and W. W. Hancock. 1980. Tissue culture of isolated glomeruli from patients with glomerulonephritis. Kidney Int. 17:515-527.

10. Thomson, N. M., S. R. Holdsworth, E. F. Glasgow, and R. C. Atkins. 1979. The macrophage in the development of experimental crescentic glomerulonephritis. Am. J. Pathol. 94:223-240.

11.Holdsworth, S. R., N. M. Thomson, E. F. Glasgow, J. P. Dowling, and R. C. Atkins. 1978. Tissue culture of isolated glomeruli in experimental crescentic glomerulonephritis. J. Exp. Med. 147:98-108.

12. Lavelle, K. J., B. D. Durland, and M. N. Yum. 1981. The effect of antimacrophage antiserum on immune complex glomerulonephritis. J. Lab. Clin. Med. 98:195-205.

13. Hunsicker, L. G., T. P. Shearer, S. B. Plattner, and D. Weisenburger. 1979. The role of monocytes in serum sickness nephritis. J. Exp. Med. 150:413-425.

14. Holdsworth, S. R., T. J. Neale, and C. B. Wilson. 1980. The participation of macrophages and monocytes in experimental immune complex glomerulonephritis. Clin. Immunol. Immunopathol. 15:510524.

15. Holdsworth, S. R., T. J. Neale, and C. B. Wilson. 1981. Abrogation of macrophage-dependent injury in experimental glomerulonephritis in the rabbit. J. Clin. Invest. 68:686-698.

16. Magil, A. B., L. D. Wadsworth, and M. Loewen. 1981. Monocytes and human renal glomerular disease: a quantitative evaluation. Lab. Invest. 44:27-33.

17. Kreisberg, J., D. Wayne, and M. Karnovsky. 1979. Rapid and focal loss of negative charge associated with mononuclear cell infiltration early in nephrotoxic serum nephritis. Kidney Int. 16:290-300.

18. Bhan, A., E. Schneeberger, A. Collins, and R. McCluskey. 1978. Evidence for a pathogenic role of a cell-mediated immune mechanism in experimental glomerulonephritis. J. Exp. Med. 148:246-260.

19. Stilmant, M., W. K. Bolton, B. C. Sturgill, and W. G. Couser. 1979. Acute crescentic glomerulonephritis without immune deposits. Clinical, serologic, and renal pathologic characteristics of 19 patients. Kidney Int. 15:184-195.

20. Bolton, W. K., and W. G. Couser. 1979. Pulse intravenous methylprednisolone therapy of acute crescentic rapidly progressive glomerulonephritis. Am. J. Med. 66:495-502.

21. Mahieu, P., M. Dardenne, and J. Bach. 1972. Detection of humoral and cell-mediated immunity to kidney basement membranes in human renal diseases. Am. J. Med. 53:185-192.

22. Macanovic, M., D. J. Evans, and D. K. Peters. 1972. Allergic response to glomerular basement membrane in patients with glomerulonephritis. Lancet. II:207-210.

23. Rocklin, R., E. Lewis, and J. David. 1970. In-vitro evidence for cellular hypersensitivity to glomerular-basement-membrane antigens in human glomerulonephritis. New Engl. J. Med. 283:497-501.

24. Fillit, H. M., S. E. Read, R. L. Sherman, J. B. Zabriskie, and I. van de Rijn. 1978. Cellular reactivity to altered glomerular basement membrane in glomerulonephritis. New Engl. J. Med. 298:861-868. 
25. Bendixen, G. 1968. Organ-specific inhibition of the in-vitro migration of leucocytes in human glomerulonephritis. Acta. Med. Scand. 184:99-103.

26. Zabriskie, J. B., R. Lewshenia, G. Moller, B. Wehle, and R. E. Falk. 1970. Lymphocytic responses to streptococcal antigens in glomerulonephritic patients. Science (Wash. DC). 168:1105-1108.

27. Janas-Boratynska, M. 1979. Cell-mediated hypersensitivity in glomerulonephritis. Arch. Immunol. Ther. Exp. 27:15-26.

28. Bolton, W. K., F. R. Benton, and B. C. Sturgill. 1978. Autoimmune glomerulotubular nephropathy in mice. Clin. Exp. Immunol. 33:463-473.

29. Bolton, W. K., F. R. Benton, and P. I. Lobo. 1978. Requirement of functional T-cells in the production of autoimmune glomerulotubular nephropathy in mice. Clin. Exp. Immunol. 33:474-477.

30. Bolton, W. K., F. L. Tucker, and B. C. Sturgill. 1980. Experimental autoimmune glomerulonephritis in chickens. J. Clin. Lab. Immunol. 3:179-184

31. Linna, T. J., D. Frommel, and R. A. Good. 1972. Effects of early cyclophosphamide treatment on the development of lymphoid organs and immunological functions in the chicken. Int. Arch. Allergy Appl. Immunol. 42:20-39.

32. Lerman, S. P., and W. P. Weidanz. 1970. The effect of cyclophosphamide on the ontogeny of the humoral immune response in chickens. J. Immunol. 105:614-619.

33. Hirota Y., and Y. Bito. 1978. The role of the thymus for maturation of transferred bursa cells into immunocompetent B cells in chickens treated with cyclophosphamide. Immunology. 35:889-899.

34. Nielsen, K. H., and R. G. White. 1974. Effect of host decomplementation on homeostasis of antibody production in fowl. Nature (Lond.). 250:234-236.

35. Bolton, W. K., N. O. Atuk, and B. C. Sturgill. 1978. Nephrotoxic nephritis in rabbits: the role of the sympathetic nervous system. Am. $J$ Pathol. 90:689-698.

36. Bolton, W. K., F. R. Benton, J. G. Maclay, and B. C. Sturgill. 1976. Spontaneous glomerular sclerosis in aging Sprague-Dawley rats. I. Lesions associated with mesangial IgM deposits. Am. J. Pathol. 85:277302.

37. Bolton, W. K., and B. C. Sturgill. 1978. Bovine serum albumin chronic serum sickness nephropathy in rats. Brit. J. Exp. Pathol. 59:167177.

38. Bolton, W. K., and R. M. Mesnard. 1982. New technique of tissue processing for immunofluorescence microscopy: formol sucrose/ gum sucrose/paraffin. Lab. Invest. 47:206-213.

39. Bolton, W. K., B. H. Spargo, and E. J. Lewis. 1974. Chronic autologous immune complex glomerulopathy: effect of cyproheptadine. J. Lab. Clin. Med. 83:695-704.

40. Mueller, J., G. Brun del Re, H. Buerki, H. U. Keller, M. W. Hess, and H. Cottier. 1975. Nonspecific acid esterase activity: a criterion for differentiation of $\mathrm{T}$ and B lymphocytes in mouse lymph nodes. Eur J. Immunol. 5:270-274.
41. Vosmík, F., I. Karakoz, J. Krejčí, J. Pekárek, T. Hraba, and K. Hála. 1975. Histological picture of the cellular response to injection of PPD into the wattle of immunized inbred chickens. Folia Biol. (Prague). 21:367.

42. Krejčí, J., I. Karakoz, J. Pekárek, T. Hraba, and K. Hála. 1974. Differences between inbred lines of chickens in the development of tuberculin hypersensitivity. Immunology. 27:133-136.

43. Snedecor, G. W., and W. G. Cochrane. 1967. Statistical Methods. Sixth edition. The lowa State University Press, Ames, Iowa.

44. Cattell, V., and S. W. Jamieson. 1978. The origin of glomerular crescents in experimental nephrotoxic serum nephritis in the rabbit. Lab. Invest. 39:584-590.

45. Dubois, C. H., J. B. Foidart, M. B. Hautier, C. A. Dechenne, M. J. Lemaire, and P. R. Mahieu. 1981. Proliferative glomerulonephritis in rats: evidence that mononuclear phagocytes infiltrating the glomeruli stimulate the proliferation of endothelial and mesangial cells. Eur. $J$. Clin. Invest. 11:91-104.

46. Unanue, E. R., and F. J. Dixon. 1965. Experimental glomerulonephritis. V. Studies on the interaction of nephrotoxic antibodies with tissues of the rat. J. Exp. Med. 121:697-714.

47. Lerner, R. A., and F. J. Dixon. 1966. Transfer of ovine experimental allergic glomerulonephritis (EAG) with serum. J. Exp. Med. 124:431-442.

48. Polverini, P. J., R. S. Cotran, M. A. Gimbrone, Jr., and E. R. Unanue. 1977. Activated macrophages induce vascular proliferation. Nature (Lond.). 269:804-806.

49. Mollo, F., O. Campobasso, M. G. Canese, G. Monga, and G. Palestro. 1977. Glomerular cell proliferation in human and experimental glomerulonephritis. Light- and electron-microscopical, and autoradiographic observations. Nephron. 18:101-108.

50. Bradfield, J. W. B., and V. Cattell. 1977. The mesangial cell in glomerulonephritis. I. Mechanisms of hypercellularity in experimental immune complex glomerulonephritis. Lab. Invest. 36:481-486.

51. Sano, M. 1976. Participation of monocytes in glomerulonephritis in acute serum sickness of rabbit. Acta. Pathol. JPN. 26:423-433.

52. Striker, G. E., M. Mannik, and M. Y. Tung. 1979. Role of marrow-derived monocytes and mesangial cells in removal of immune complexes from renal glomeruli. J. Exp. Med. 149:127-136.

53. Shigematsu, H., and Y. Kobayashi. 1976. Accelerated serum sickness in the rabbit. II. Glomerular ultrastructural lesions in transient proliferative and progressive disorganizing glomerulonephritis. Virchows Arch. A Pathol. Anat. Histol. 369:269-282.

54. Schiffer, M. S., and A. F. Michael. 1978. Renal cell turnover studied by $\mathrm{Y}$ chromosome ( $\mathrm{Y}$ body) staining of the transplanted human kidney. J. Lab. Clin. Med. 92:841-848.

55. Brune, K., and J. K. Spitznagel. 1973. Peroxidaseless chicken leukocytes: isolation and characterization of antibacterial granules. $J$. Infect. Dis. 127:84-94. 